\title{
Lumen
}

Selected Proceedings from the Canadian Society for Eighteenth-Century Studies

\section{Pratiques cartographiques en Nouvelle-France}

La prise en charge de l’État dans la description de son espace

colonial à l'orée du XVIII ${ }^{\mathrm{e}}$ siècle

\section{Jean-François Palomino}

Volume 31, 2012

URI : https://id.erudit.org/iderudit/1013065ar

DOI : https://doi.org/10.7202/1013065ar

Aller au sommaire du numéro

Éditeur(s)

Canadian Society for Eighteenth-Century Studies / Société canadienne d'étude du dix-huitième siècle

ISSN

1209-3696 (imprimé)

1927-8284 (numérique)

Découvrir la revue

Citer cet article

Palomino, J.-F. (2012). Pratiques cartographiques en Nouvelle-France : la prise en charge de l'État dans la description de son espace colonial à l'orée du XVIII ${ }^{\mathrm{e}}$ siècle. Lumen, 31, 21-39. https://doi.org/10.7202/1013065ar

Copyright (C Canadian Society for Eighteenth-Century Studies / Sociéte canadienne d'étude du dix-huitième siècle, 2012
Ce document est protégé par la loi sur le droit d'auteur. L'utilisation des services d'Érudit (y compris la reproduction) est assujettie à sa politique d'utilisation que vous pouvez consulter en ligne.

https://apropos.erudit.org/fr/usagers/politique-dutilisation/ 


\title{
Pratiques cartographiques en Nouvelle-France :
}

\author{
la prise en charge de l'État dans la description \\ de son espace colonial à l'orée du XVIII ${ }^{\mathrm{e}}$ siècle
}

Jean-François Palomino

Bibliothèque et Archives nationales du Québec

Le présent article s'inscrit dans une enquête plus large sur le rôle des savoirs géographiques en milieu colonial, plus spécifiquement en Nouvelle-France ${ }^{1}$. Il se veut une présentation des principaux enjeux cartographiques accaparant l'État français à l'orée du XVIII e siècle. Faut-il y voir les effets d'une meilleure organisation archivistique sous la férule de Colbert? Toujours est-il que les années 1680 sont particulièrement fastes en matière de cartographie. En effet, les archives coloniales regorgent de cartes du Canada datant de cette période, durant laquelle des professionnels aguerris à l'art du dessin jettent les bases d'une nouvelle cartographie appuyée par l'État. Ils se nomment Villeneuve, Deshayes, Jolliet et Franquelin et, tous ensemble, couvrent l'essentiel de la cartographie pratiquée en Nouvelle-France: topographie, hydrographie, description continentale. D'autres avant eux se sont adonnés à cet art, mais pas de façon aussi systématique. Lun d'entre eux, le géographe du roi Jean Baptiste Louis Franquelin, expose d'ailleurs assez clairement ces enjeux dans deux mémoires présentés aux secrétaires d'État à la Marine. Dans le premier mémoire, datant de 1689, Franquelin suggère d'explorer, de cartographier et de

1. La plupart des cartes traitées dans le présent article sont reproduites, en tout ou en partie, dans La mesure d'un continent -Atlas historique de l'Amérique du Nord, 1492-1814, Sillery, Septentrion, 2007. 
tracer les limites de l'empire français pour empêcher l'ennemi de s'y installer et d'en contrôler les voies d'accès. Il propose d'envoyer, au sud ou au nord, « une personne qui puisse tirer des lignes justes et former des limittes en plantant des bornes avec les armes de Sa Majesté ${ }^{\star}$. Trois ans plus tard, après avoir perdu sa femme et plusieurs de ses enfants dans un naufrage sur le fleuve Saint-Laurent, Franquelin rédige un vaste programme cartographique décliné en plusieurs volets ${ }^{3}$ :

1. Cartographier les terres défrichées, les habitations, les concessions et les seigneuries conformément au cadastre.

2. Explorer l'intérieur du continent pour repérer les endroits propices à l'exploitation.

3. Tracer des chemins entre Québec, l'Acadie et les colonies anglaises.

4. Faire des plans en relief de Québec et d'autres endroits stratégiques.

5. Chercher le plus court chemin pour aller à la baie d'Hudson.

6. Cartographier le golfe et le fleuve Saint-Laurent afin que la navigation y soit plus sûre et plus aisée, tout en identifiant les bons endroits de pêche pour y faire des établissements solides.

$\mathrm{Si}$, dans leur ensemble, ces propositions apparaissent relativement ambitieuses par rapport aux moyens alors consentis par l'État, elles permettent néanmoins de cerner les enjeux d’ordre géographique qui occupent les Français au début du XviII ${ }^{\mathrm{e}}$ siècle, alors que l'État se construit et cherche à mieux administrer son espace colonial. Ces propositions ne sont pas seulement le fruit d'un homme désemparé cherchant une occupation pour oublier son malheur; il faut surtout les voir comme le reflet de ce qui est concrètement envisageable et de ce qui est localement nécessaire. Reste à savoir jusqu’à quel point l'État central est en mesure d'investir les sommes nécessaires pour en assurer la réalisation.

2. J. B. L. Franquelin, Mémoire pour informer Monseigneur de l'importance qu'il $y$ a de tirer de lignes justes sur les limites des terres qui appartiennent au Roy dans la Nouvelle France, planter des bornes, arborer des armes de sa Majesté, et en faire une carte bien fidelle, [1689]. Bibliothèque nationale de France $(\mathrm{BnF}), \mathrm{Mss}$. Occ., Clairambault, vol. 879, fol. 289 .

3. J. B. L. Franquelin, Memoire touchant les voyages que Franquelin, hydrographe du roy en Canada, a faits de Québec à Paris pour le service de Sa Majesté, 1693. BnF, Mss. Occ., Clairambault, vol. 879, fol. 294. 


\section{Cartographie topographique et planification géostratégique}

«Voici M"gr l'un des dessineurs que j'ay eu l'honneur de vous proposer. [...] Du surplus il est capable de bien lever les plans et profils de quelque lieu que ce soit et d'assez bien faire une carte qui est a mon avis tout ce qui peut vous faire besoing au pais ou vous voulez l'envoyer. » Écrivant au ministre Seignelay, le commissaire des fortifications Vauban poursuit ainsi :

$\mathrm{M}^{\mathrm{r}}$ Denonville pourra fort bien vous mander les raisons qu'on peut avoir de faire des establissements dans les scituations plus importantes et d'y bastir aussy bien que les commodités qui pouroient favoriser ses pensées, auquel cas en vous en envoyant des plans et profils je vous en feray des desseins a peu près comme si j'etais sur les lieux. [...] Il est assez joly garcon et dessine assez habilement. Je ne doute pas que $\mathrm{M}^{\mathrm{r}}$ Denonville ne s'en serve fort utilement ${ }^{4}$.

L'ingénieur dont il est question dans l'extrait ci-dessus promet d'être un excellent dessinateur. Tant mieux si, en plus, il est joli garçon... Recommandé par Vauban, Robert de Villeneuve est sur-le-champ engagé par le ministre Seignelay, qui lui ordonne de «partir incessamment [...] à La Rochelle» pour y rejoindre Denonville, fraîchement nommé gouverneur de la Nouvelle-France 5 . Dès son arrivée dans la colonie, en mai 1685, Villeneuve s'attelle à la tâche, cartographiant tout d'abord la ville et le château de Québec ${ }^{6}$, puis, malgré la maladie qui l'afflige, Trois-Rivières, Montréal et Cataracoui. L'année suivante, il cartographie l'île d'Orléans ainsi que la «tête de la colonie », c'est-àdire Ville-Marie, Chambly, la Prairie-de-la-Magdelaine, la rivière des Prairies et la seigneurie de la Chesnaye (Lachenaie). Il s'agit, pour le gouverneur, de bien montrer au ministre la nécessité d'occuper ces terres avec des troupes pour sécuriser le pays ${ }^{7}$. La correspondance officielle souligne l'importance géostratégique de Chambly, qui

4. Lettre de Vauban à Seignelay, 10 mars 1685. Cité dans La correspondance de Vauban relative au Canada, documents présentés par Louise Dechêne, [Québec], ministère des Affaires culturelles, 1968, p. 9.

5. Lettre de Seignelay à Vauban, 2 avril 1685. Cité dans La correspondance de Vauban relative au Canada, op. cit., p. 10.

6. Robert de Villeneuve, Plan de la ville et chasteau de Québec, [Québec], 1685. Archives nationales d'outre-mer (ANOM), Aix-en-Provence, $3 \mathrm{DFC}_{349} \mathrm{~B}$.

7. Lettre de Denonville à Seignelay, 10 novembre 1686. ANOM, COL CiıA 8 / fol. 129-159. 
contrôle l'accès au lac Champlain, l'une des principales voies de communication vers les colonies anglaises au sud. En 1689, Villeneuve cartographie l'île d'Orléans; l'année suivante, il dresse une carte de Québec assiégé par les Anglais8.

Lun des premiers ingénieurs à séjourner au Canada, Villeneuve accomplit un travail remarquable eu égard aux moyens dont il dispose. Il dresse des cartes d'une précision inégalée par rapport à tout ce qui s'était réalisé jusqu'alors. Ses œuvres renseignent sur les routes, les rivières, les forêts, les terres défrichées et cultivées, les habitations, le relief. Par son habileté à dessiner fidèlement les lieux observés, par son usage et sa maîtrise des codes picturaux appropriés, Villeneuve devait permettre à Vauban de connaître le pays à distance, de s'approprier la topographie, de repérer les voies d'accès par où les ennemis anglais et iroquois pouvaient attaquer. Une excellente connaissance du terrain était nécessaire pour fortifier et aménager les villes tout en assurant la sécurité des colons.

Pour instruire Sa Majesté de l'état des lieux, Villeneuve propose même de faire des plans en relief de Québec, de Trois-Rivières et de Montréal, sans toutefois que sa proposition ait de suite immédiate. Il n'empêche que Vauban demandera et obtiendra des appointements de 1200 livres par année pour son protégé'. Cette somme est l'une des dépenses salariales les plus importantes dans la colonie si l'on exclut les gages des principaux dirigeants. Malgré cela, le gouverneur Denonville éprouve quelque difficulté à mettre l'ingénieur du roi à sa main, le qualifiant notamment de «fol, libertin et débauché». Il lui reproche surtout un caractère indocile, se félicitant que les réprimandes adressées par Vauban l'aient rendu " plus souple et plus traitable ${ }^{10}$ ». Malgré des relations houleuses avec les autorités en place, Villeneuve demeure six ans au Canada. S'il peut tenir tête aux autorités, c'est qu'il jouit d'un rapport de force favorable, aucun autre ingénieur ne lui faisant concurrence. D'ailleurs, Denonville reconnaîtra constamment

8. Robert de Villeneuve, Plan de Québec et de ses environs, en la Nouvelle-France, assiégé par les Anglais, 1690. BnF, CPL GeD-8053 et GeDD-2987 (8674).

9. «1200 tt d'appointements et quelque chose pour ses voyages quand on luy en fera faire, c'est a mon advis ce qu'il luy faudroit donner. » Vauban à Seignelay, 10 mars 1685. Cité dans La correspondance de Vauban relative au Canada, op. cit., p. 9.

10. Lettre de Denonville et Champigny à Seignelay, 6 novembre 1688. ANOM, COL Ci1A 10 / fol. 8-16v. 
son talent et l'utilité de son travail. Après son départ définitif, en novembre 1692, d'autres ingénieurs vont poursuivre son œuvre cartographique avec succès, assurant une planification géostratégique ainsi qu'une communication transatlantique relativement continue jusqu'à la chute de la Nouvelle-France: qu'on pense au sieur Levasseur, au sieur Beaucours ainsi qu'à Gaspard-Joseph Chaussegros de Léry, le plus prolifique des ingénieurs du Canada, actif depuis son arrivée, en 1716, jusqu'à sa mort, en 1756. Ce dernier envoie une centaine de plans depuis Québec vers la métropole, notamment des plans en relief de Québec et de Montréal, permettant de faire connaître au roi les enjeux urbanistiques propres à chacune des villes et proposant pour approbation des plans de construction et de réparation des fortifications et d'autres bâtiments publics. Les connaissances des ingénieurs sont d'une telle importance qu'on se propose parfois même de les tuer pour éviter qu'ils ne transmettent des renseignements cruciaux à l'ennemi ${ }^{11}$ !

D’autres ingénieurs et dessinateurs de talent vont également représenter les villes coloniales du Canada et de la Louisiane, que ce soit Labat en Acadie, L'Hermitte à Terre-Neuve ou Le Blond de La Tour, Pauger, Broutin, Devin et Devergès en Louisiane. Lorsqu'en 1713 les Français doivent abandonner Port-Royal et Plaisance et repenser le déploiement de la population et des forces armées du royaume, plusieurs hommes d'expérience et de talent sont mobilisés pour repérer les endroits les plus propices à de nouveaux établissements, notamment Verville, L'Hermitte, de Couagne, Boishébert et Beaucours. On explore et cartographie des côtes peu connues, on évalue la qualité des bois et des terres ainsi que la possibilité d'établir de nouvelles pêcheries. Une pléthore de cartes et de mémoires sont déposés pour fins d'étude, permettant aux autorités ${ }^{12}$ d'effectuer un choix éclairé. La décision est d'importance: la France s'autorise un nouveau départ et des sommes importantes sont investies à cet effet. Les côtes du Labrador, de l'île Saint-Jean et de l'île Royale sont examinées en détail avant qu'on ne

11. Après avoir quitté le Canada, Villeneuve est envoyé dans le Dauphiné, par mesure disciplinaire semble-t-il. Écrivant au directeur des fortifications à son sujet, Vauban se propose de le faire tuer, car il «en sait assez pour pouvoir nuire». Voir Michèle Virol, Vauban: de la gloire du roi au service de l'État (Éditions Champ Vallon, 2003, p. 40), pour qui Vauban recourt à l'ironie en écrivant ces lignes. On perd ensuite la trace de l'ingénieur, qui ne donne plus signe de vie...

12. Notamment le tout nouveau conseil de Marine mis en place par le duc d'Orléans en 1715 . 
retienne l'emplacement du Havre-à-l'Anglais, où l'on fait construire une véritable forteresse portuaire: Louisbourg. En protégeant ce lieu libre de glace à longueur d'année, on favorise la communication avec la métropole et on contrôle l'entrée du Saint-Laurent tout en soutenant l'industrie de la pêche.

On s'en doute, le corps des ingénieurs fut d'une importance capitale pour le développement des colonies, tout comme leur maîtrise du langage cartographique le fut pour faire valoir leurs projets. Correspondant directement avec le ministre et non pas seulement par le truchement des autorités locales, ils ont droit à toute l'attention nécessaire. Ils sont de ceux qui obtiennent le plus souvent des gratifications ou des primes pour services rendus, leur savoir-faire étant hautement prisé. Sans conteste, le poste d'ingénieur est indispensable aux administrateurs de la colonie. Il le demeurera jusqu'à la chute de la Nouvelle-France, en $1760^{13}$.

\section{L'hydrographie du fleuve Saint-Laurent}

Pendant que Villeneuve parcourt les campagnes du Canada avec ses instruments de travail, d'autres cartographes s'activent également sur les eaux du fleuve Saint-Laurent. À l'époque, écueils, rochers, battures, courants, brouillards et tempêtes engloutissent plus d'un navire, causant la perte d'un bon nombre de vies humaines. Dès son arrivée au Canada, le gouverneur Denonville remarque que les cartes (d'origine hollandaise) utilisées par les navigateurs ne valent rien ${ }^{14}$. Après avoir suggéré de faire appel aux services du pilote Chaviteau, il recommande chaudement le commerçant Louis Jolliet (1645-1700), seigneur de Mingan et d'Anticosti, né au Canada, «homme de bien [qui] pourroit instruire et former des pilottes en ce pays ${ }^{15} »$. Dans une lettre envoyée au secrétaire d'État de la Marine, Jolliet écrit: «Ce n’est pas sans raison que de tout temps, ceux qui sont venus dans ce pays de la Nouvelle-

13. Pour un portrait détaillé de plusieurs des ingénieurs nommés, voir le Dictionnaire biographique du Canada (www.biographi.ca) ainsi que Laurent Vidal et Émilie d'Orgeix, (édit.), Les villes françaises du Nouveau Monde - Des premiers fondateurs aux ingénieurs du roi (XVI ${ }^{e}$-XVIII $I^{e}$ siècles), Paris, Somogy, 1999.

14. Lettre de Denonville à Seignelay, 20 août 1685. ANOM, COL C11A 7 / fol. 55 -6ov.

15. Lettre de Denonville à Seignelay, 13 novembre 1685 . ANOM, COL C11A 7 / fol. 86-106v. 
France, ont appréhendé l'entrée du golfe de Saint-Laurent et tous les passages depuis Anticosti jusqu'à Québec, à plus de cent trente lieues l'un de l'autre. On sait, Monseigneur, que plusieurs navires envoyés par Sa Majesté aussi bien que par les marchands ont péri dans ledit fleuve, faute de cartes sur lesquelles on peut naviguer ${ }^{16}$. » Ces quelques lignes permettent à Jolliet d'introduire son immense carte de l'estuaire du Saint-Laurent depuis Québec jusqu'à Terre-Neuve, achevée après 46 voyages en barque et en canot $^{17}$. Cette carte ne porte pas la mesure de profondeur des eaux, mais elle comporte les caps, îles, battures et mouillages ainsi que les trajets à suivre pour éviter les récifs et les hauts-fonds ${ }^{18}$.

Au même moment, le pays reçoit la visite d'un invité de marque: le mathématicien Jean Deshayes, envoyé par l'Académie royale des sciences. Fondée en 1666, cette institution s'était donné pour mission de redessiner la carte du monde selon des mesures plus précises tirées d'observations astronomiques. Le Canada n'est pas la première des destinations exotiques de Deshayes. Trois ans plus tôt, il s'était rendu à Gorée, puis en Guadeloupe et en Martinique, avec une équipe de savants français qui s'aventuraient alors vers des terres outre-mer pour y mener leurs expérimentations ${ }^{19}$, préfigurant ainsi les grandes expéditions scientifiques du siècle des Lumières. Éditeur scientifique de deux livres techniques, l'un sur le compas de proportion ${ }^{20}$ et l'autre sur le nivellement ${ }^{21}$, Deshayes maîtrisait les instruments nécessaires aux prises de mesures. C'est donc un candidat de choix qui s'amène à

16. Lettre de L. Jolliet à Seignelay, 10 novembre 1685. ANOM, COL C11E 13 / fol. $135-136$.

17. Carte du grand fleuve St Laurens dressée et dessignée sur les memoires \& observations que le $s^{r}$ Jolliet a très exactement faites en barq: 6 en canot en 46 voyages pendant plusieurs années, 1685. BnF, Ge SH 126-1-3(1) Rés.

18. Dix ans plus tard, en novembre 1695 , le capitaine du navire La Charente, craignant de devoir affronter le Saint-Laurent en saison aussi avancée, demande l'aide d'un pilote expérimenté. Louis Jolliet est alors désigné pour conduire ce navire chargé de fourrures jusqu'en France. Là-bas, on lui octroie le titre d'hydrographe du roi, qu'il porte jusqu'à sa mort, en 1700.

19. Nicholas Dew, «Scientific travel in the Atlantic world: the French expedition to Gorée and the Antilles, 1681-1683», The British Journal for the History of Science, 43, 1, 2010, p. 1-17.

20. L'Usage du compas de proportion de D. Henrion, nouvellement revû, corrigé é augmenté en toutes ses parties de plusieurs propositions nouvelles et utiles, par le sieur Deshayes, professeur és Mathematiques, Paris, 1681.

21. Jean Deshayes, La Théorie et la pratique du nivellement, Paris, l'auteur, 1685. 
Québec en 1685 pour effectuer les relevés précis du fleuve SaintLaurent. À l'automne, malgré un état de santé «moribond ${ }^{22}$ », Deshayes accompagne le gouverneur Denonville jusqu'au lac Ontario pour «prendre les plans de trois petites places qui sont sur cette rivière, les Trois Rivières, Montreal et Cataracouy ${ }^{23} »$. Ce voyage aller-retour de plus de $1000 \mathrm{~km}$ lui permet de dresser une carte du fleuve en amont de Québec, «a veuë sur la simple boussole et sur l'estime du che$\min ^{24}$ ». Le 10 décembre de la même année, l'observation approximative d'une éclipse de lune comparée à l'observation de la même éclipse à Paris permet de calculer la longitude de Québec avec une précision inégalée pour l'époque ${ }^{25}$. Durant l'hiver, Deshayes parcourt la côte sud (jusqu'à Rivière-Ouelle) et l'île d'Orléans en raquettes, comptant chacun de ses pas pour calculer la distance parcourue. Lannée suivante, équipé d'une barque et d'un canot d'écorce, il cartographie et sonde l'estuaire du Saint-Laurent avec l'aide de quelques matelots. Longeant la côte nord, de Québec jusqu'à Sept-Îles, il met en application ses connaissances étendues en mathématiques et triangulation pour dessiner le rivage. Il sonde également le fleuve pour en connaître la profondeur, tracer des chenaux et désigner les lieux de mouillage, bref, pendant une période d'au moins cinq mois, Deshayes s'évertue à colliger toutes les données utiles à la navigation sur le fleuve. L'ensemble de sa démarche scientifique est d'ailleurs détaillé dans un recueil manuscrit, conservé parmi les archives du Séminaire de Québec ${ }^{26}$. On

22. «Je l'ay mene tout moribond avec moy a mon voyage de Catarok8y pendant lequel je luy ay fait tracer notre Riviere d'icy au lac Ontario, non sans peine car je croyais a tout moment qu'il moureroit dans mon canot. » Lettre de Denonville à Seignelay, 13 novembre 1685. ANOM, COL C11A 7 / fol.86-106v.

23. Jean Deshayes, Carte marine de la Riviere de Quebec par le Sr Deshayes, 1686, ou Recueil de ce qui sert a la navigation particuliere de cette riviere et de ce qui peut contribuer a la metode generale de lever et dresser les cartes marines, entre 1692 et 1706. Musée de la civilisation, fonds d'archives du Séminaire de Québec. Polygraphie 2, $\mathrm{n}^{\circ} 34$, fol. 13 .

24. Ibid.

25. Voir Jean Deshayes, De la Grande Rivière de Canada appellée par les Européens de St. Laurens, Paris, Nicolas de Fer, 1715. BAnQ, G 3312 S 51715 D4. "Par une observation faitte sans instrumens a Quebec de l'eclipse de lune du 10 decembre 1685 comparée par $\mathrm{M}^{\mathrm{r}}$ Cassini a celle de Paris. On a trouvé la difference de longitude de Paris a Quebec occidentale 4 heures 48 minutes 52 secondes. »

26. Jean Deshayes, Carte marine de la Riviere de Quebec par le $S^{r}$ Deshayes. 1686, ou Recueil de ce qui sert a la navigation particuliere de cette riviere..., op. cit. Pour une excellente mise en contexte du recueil, voir James S. Pritchard, «Early French 
y perçoit bien toutes les difficultés qu'il y avait de cartographier un vaste territoire, peu habité, difficile à parcourir été comme hiver.

Malgré l'ampleur du travail qu'il reste à accomplir, notamment cartographier le golfe du Saint-Laurent, Deshayes rentre au bercail dès l'année suivante sans qu'on sache exactement pourquoi. Il retournera à Québec 16 ans plus tard comme hydrographe du roi pour diriger une école d'hydrographie l'hiver et poursuivre la cartographie du fleuve l'été, plus particulièrement sur la côte du Labrador, de plus en plus fréquentée par les marchands français installés dans la colonie ${ }^{27}$. Son salaire annuel s'élève alors à 800 livres, montant moindre que celui d'un ingénieur mais plus élevé que ce que gagnaient la plupart des autres salariés de l'État, par exemple le chirurgien, l'armurier, le gardemagasin, le canonnier, etc. ${ }^{28}$.

L'héritage le plus précieux de Deshayes est sans conteste sa cartographie du fleuve, d'une précision telle qu'on peut y apercevoir maisons et moulins donnant sur le fleuve. Après que l'Académie royale des sciences l'eut jugée d'une grande utilité pour la navigation, son œuvre cartographique fut publiée à échelle réduite, probablement vers 1700, avant d'être rééditée en $1715^{29}$, avec un texte détaillé fourmillant d'indications à l'intention des navigateurs: repères, direction, nature et

Hydrographic Surveys in the Saint Lawrence River ", International Hydrographic Review, 56, 1, 1979, p. 125-142.

27. En 1704, il dresse une carte de l'établissement de M. de Courtemanche, sur la côte du Labrador, qu'il transmet à l'intendant Raudot (Coste du Canada depuis Quégasca, pays des Esquimaux, jusqu'a la riviere Quesesasquiou, côte de Labrador. $\mathrm{BnF}, \mathrm{Ge} \mathrm{SH}$ 124-3-8). Au sujet de cette carte, voir le résumé d'une lettre de Deshayes datée du 18 octobre 1705. ANOM, COL C11A 23 / fol. 198v-199v.

28. État des fonds à faire pour les dépenses de la Nouvelle-France pendant l'année 1707 - Appointements des officiers généraux. ANOM, COL C11A 113 / fol. 211$213 \mathrm{~V}$.

29. Deshayes mentionne avoir produit plusieurs esquisses préparatoires, dont l'une est présentée sans délai au marquis de Seignelay. Sa première carte manuscrite est fort probablement celle intitulée Carte du cours du fleuve de S. Laurent depuis Québec jusqu'au lac Ontario, [1685?], conservée au Service historique de la Défense (SHD), rec. 67, n 83. Une autre carte manuscrite, intitulée Carte de la Riuere de St Laurens leuee sur les lieux en 1686, se trouve dans la collection du Service hydrographique de la $\mathrm{BnF}$ à la cote Ge SH 126-1-4. La première édition de la carte imprimée intitulée Carte marine de l'embouchure de la rivière de $\mathrm{S}$. Laurens, levée de cap en cap jusqu'à Québec (sans date et sans mention d'éditeur) se trouve également à la $\mathrm{BnF}$ (GE DD-2987 (8658 B)). Pour voir la réédition de 1715 (Jean Deshayes, De la Grande Rivière de Canada appellée par les Européens de St. Laurens, Paris, Nicolas de Fer), voir l'exemplaire numérisé sur le site de BAnQ (G $\left.3312 \mathrm{~S}_{5} 1715 \mathrm{D}_{4}\right)$. 
profondeur des fonds, lieux de mouillage, éloignement souhaitable de la côte, battures, écueils. Près de 80 ans plus tard, le cartographe officiel du Dépôt des cartes et plans de la Marine jugera cette carte d'une grande utilité en raison de ses détails et de son exactitude ${ }^{30}$. Non longtemps avant sa mort, Deshayes propose de parcourir la NouvelleFrance, depuis Plaisance jusqu'à Détroit, afin d'établir une nouvelle cartographie basée sur des mesures fixes qui auraient permis de corriger les cartes de ses prédécesseurs en lesquelles il n'avait pas confiance ${ }^{31}$. Pour réaliser ce projet, il supplie les autorités de lui fournir un pendule afin de pouvoir calculer la longitude de différents endroits clés, aux marges de la colonie, soit Plaisance à l'est et Détroit à l'ouest. Malheureusement, la mort l'empêche de mettre ce projet à exécution. Néanmoins, la fonction d'hydrographe du roi et de maître d'hydrographie est alors bien établie dans la colonie. Au décès de Deshayes, les Jésuites convoitent et obtiennent le poste laissé vacant. Il sera occupé par plusieurs de leurs membres, notamment par le père Bonnécamps, certainement l'un des savants français les plus actifs au Canada au XVIII $^{\mathrm{e}}$ siècle. Jusqu'à la chute de la Nouvelle-France, divers cartographes sillonnent le fleuve Saint-Laurent et son golfe pour lever de nouvelles cartes, qu'on veut toujours plus précises. Certains endroits stratégiques sont plus particulièrement visés: Kamouraska et RivièreOuelle $^{32}$, où l'on pêche le béluga, dont le lard est transformé en un précieux combustible pour l'éclairage; le Labrador ${ }^{33}$, où l'on chasse le loup marin et où l'on pêche la morue; les côtes de Terre-Neuve, avant qu'elles ne soient cédées à l'Angleterre en 1713; le détroit de Belle-Isle, passage stratégique s'il en est un; enfin, l'île de Sable, dont on ne connaît pas l'emplacement exact, véritable bête noire des navigateurs, à l'origine de nombreux naufrages ${ }^{34}$.

30. Attribuée à Jacques Nicolas Bellin, Exposition des observations et données d'après lesquelles a été imprimée la carte du golfe et fleuve Saint-Laurent-Description des côtes, baies, havres, etc., [s. d.]. ANOM, COL C11E 13 / fol. 75-10ov.

31. Résumé d'une lettre de J. Deshayes, 18 octobre 1705. ANOM, COL C11A 23 / fol. $198 \mathrm{v}-199 \mathrm{v}$.

32. Voir notamment plusieurs plans de L. Peyre, vers 1728. BAnQ, P6oo, $\mathrm{S}_{4}, \mathrm{SS}_{2}$, D24-36.

33. Voir par exemple François Desmoulins de Beauvilliers, Carte particulière depuis la riviere des Equimaux jusqu'à la pointe de Belsamont, 1715. Newberry Library, Ayer MS map 30 sheet 100.

34. Voir notamment le projet du sieur de La Brosse de lever une carte du SaintLaurent présenté au Conseil de Marine: «Le principal objet de la levée de cette carte 


\section{Jean Baptiste Louis Franquelin et la cartographie continentale}

Lorsque Deshayes quitte le Canada après son premier séjour, il laisse vacant un nouveau poste jugé utile que les autorités s'empressent de pourvoir. Celles-ci choisissent un excellent dessinateur en la personne de Jean Baptiste Louis Franquelin ${ }^{35}$. Arrivé dans la colonie en ${ }^{1672}$, Franquelin avait été sollicité pour dessiner le fleuve Mississippi « découvert» par Jacques Marquette et Louis Jolliet. Engagé de façon ponctuelle pendant plusieurs années, il peut, une fois nommé officiellement hydrographe du roi, se consacrer entièrement à l'enseignement et à la cartographie, recevant dorénavant des gages sur une base régulière. Mais les intérêts de Franquelin débordent le strict domaine de l'hydrographie puisqu'il dessine, tout au long de sa carrière, une grande variété de cartes qui témoignent des besoins de l'État. Sa production aborde tout autant le plan urbain que la carte du fleuve, du continent et des territoires ennemis. Les cartes et autres documents d'archives rappellent que Franquelin cartographie "par l'ordre des gouverneurs et intendants du pays, pour le service du Roy, et pour leur instruction particulière, et suivant les memoires et relations qu'il a eu soin de recueillir exactement par le mesme ordre \& pendant le mesme temps, de tous les voyageurs les plus entendus, qu'il a consultez et confrontez avec une application toute particuliere ${ }^{36}$ ». Établi à Québec, Franquelin a pu intercepter ceux qui revenaient de l'Ouest et accéder à leurs journaux de voyage lorsqu'ils en avaient. Il a pu rencontrer les plus célèbres explorateurs de la Nouvelle-France: non seulement Jolliet, LaSalle, d'Iberville, Cadillac, Dulhut et Hennepin mais certainement d'autres moins connus aussi. Franquelin a pu les interroger en personne, instaurer avec eux un dialogue (plus difficile, voire impossible à instaurer pour le cartographe européen). Il a donné une voix aux voyageurs illettrés, il a couché sur papier leurs parcours, leurs lieux de

doit estre la decouverte de l'isle de Sable dont la latitude et la longitude ne sont connues que par les observations peu justes de ceux qui y ont fait naufrage et s'en sont sauvés sur le debris de leurs vaisseaux. » ANOM, COL C11A 37 / fol. 46-49.

35. Voir les remerciements de Franquelin à Seignelay pour l'emploi d'hydrographe du roi à Québec, 5 novembre 1686. BnF, Mss. Occ., Clairambault, vol. 879, fol. 285 .

36. J. B. L. Franquelin, Carte de l'Amerique septentrionnalle, Québec, 1688. SHD, rec. $66, n^{\circ} 6$ bis. 
passage. Sous sa plume, de nouveaux lacs, de nouvelles rivières, de nouveaux peuples amérindiens ont été portés à la connaissance des Européens ${ }^{37}$.

Parmi ses grandes réalisations, on retient une carte dressée en 1688 à la demande de Louis XIV, qui voulait mieux connaître les limites de son empire en Amérique du $\operatorname{Nord}^{38}$. Franquelin fait le voyage depuis Québec jusqu'à Versailles pour présenter lui-même son œuvre, qui donne à voir non seulement de nouvelles découvertes géographiques mais aussi des particularités ornementales bien originales, en l'occurrence des armoiries du roi symbolisant l'appropriation du territoire par les Français, de même qu'une splendide vue de Québec, minutieusement dessinée, qui permet de faire connaître au roi l'aspect physique de la capitale de son royaume en Amérique du Nord.

Ailleurs, le cartographe croque des Amérindiens, nouveaux sujets du roi, dans différentes postures, les uns cuisinant, les autres fumant la pipe ou tenant une règle à mesurer. Il s'agit pour Franquelin de transmettre non seulement des connaissances géographiques mais aussi des connaissances sur les habitants des lieux, sur sa faune et sur sa flore. Loin d'être marginales, les illustrations en marge contribuent au subtil message que les représentants du roi (gouverneurs et intendants locaux) veulent communiquer au souverain, qui consiste à attirer son attention pour obtenir les fonds nécessaires au développement de la colonie.

Non seulement Franquelin met constamment à jour sa carte du continent au fil des nouvelles explorations, il dessine aussi les territoires ennemis. Vers 1687, il dessine le territoire des Iroquois, dans la mire du gouverneur Denonville qui n'en souhaite rien de moins que la «destruction totale ${ }^{39} »$. Franquelin signale les cours d'eau, le relief, l'emplacement des villages, le nombre de cabanes qui les composent et les chemins qui les relient les uns aux autres, des données toutes utiles à une expédition militaire.

37. Sur Franquelin et son œuvre, voir notamment Jean Delanglez, «Franquelin, Mapmaker », Mid-America, 25, 1943, p. 29-74, et Jean-François Palomino, «Un géographe du roi à Québec à la fin du XVII siècle : Jean-Baptiste Franquelin », dans Les villes françaises du Nouveau Monde, op. cit., p. 162-167.

38. J. B. L. Franquelin, Carte de l'Amerique septentrionnalle, Québec, 1688. SHD, rec. $66, \mathrm{n}^{\circ} 6$ bis.

39. J. B. L. Franquelin, Carte du Pays des Irroquois, vers 1687. SHD, rec. 67, n 66. Comme cette carte n'est pas datée, il est difficile de savoir si elle constitue le plan préparatoire ou l'aboutissement de l'expédition militaire de Denonville. 
Quelques années plus tard, alors que Français et Anglais se disputent ouvertement leurs territoires coloniaux, Franquelin est envoyé par le gouverneur Frontenac en mission d'espionnage, accompagné de Lamothe de Cadillac. Car pour le roi, il n'y a aucun doute permis: seule une attaque définitive sur Manhattan peut mettre un terme à une guerre désastreuse pour la colonie. Franquelin doit rapporter les cartes des ports, des havres et des villes principales de la NouvelleAngleterre. Dans un délai de cinq mois, il parvient à dessiner des plans relativement précis pour les circonstances ${ }^{40}$, peaufinant son tracé jusqu'à ce que tous ceux qui avaient été sur les lieux, Français, Anglais ou Amérindiens, les aient approuvés. Ces cartes mettent en valeur les sondes et autres observations utiles pour le mouillage des vaisseaux et l'abordage des terres ainsi que les routes qui mènent à la ville, par terre et par mer. Elles vont d'ailleurs servir de sources à Jacques Nicolas Bellin, qui les reproduit presque fidèlement pour son Petit atlas maritime 70 ans plus tard.

En tant que géographe du roi, Franquelin n'eut pas de véritable successeur au Canada, sauf peut-être Jean Baptiste de Couagne, qui dessina quelques cartes à l'échelle continentale, notamment pour exposer les enjeux frontaliers aux négociateurs du traité d'Utrecht ${ }^{41}$. En effet, avec la création officielle du Dépôt des cartes et plans de la Marine en 1720, le centre de gravité du traitement des données se déplace outre-mer, de Québec vers la métropole. Le processus de centralisation des savoirs se fait encore plus marqué. Dans la métropole, l'hydrographe et ingénieur Jacques Nicolas Bellin s'occupe de recueillir, de mettre en ordre et d'exploiter les cartes qu'il reçoit; autant archiviste que cartographe, il s'avérera un cartographe plus que prolifique ${ }^{42}$.

40. Au moins trois cartes de Franquelin sont issues de cette expédition, conservées à la $\mathrm{BnF}(\mathrm{Ge} \mathrm{SH}$ 135-1-1; Ge SH 135-6-6 et Ge SH 135-6-7).

41. Jean-Baptiste de Couagne, Carte du Canada ou les terres des François sont marquées de bleu et celle des Anglois de jaune, 1712. SHD, rec. 67, $\mathrm{n}^{\circ} 13$.

42. Sur Bellin, voir notamment Jean-François Palomino, «Entre la recherche du vrai et l'amour de la patrie : cartographier la Nouvelle-France au XVIII siècle ", Revue de Bibliothèque et Archives nationales du Québec, 1, 2009, p. 84-99. Les cartes du Dépôt des cartes et plans font maintenant partie intégrante de la collection du Service hydrographique de la Marine, scindée entre la Bibliothèque nationale de France, le Service historique de la Défense à Vincennes et les Archives nationales de France. 


\section{Aux confins du continent: les voyageurs cartographes}

Si les cartographes professionnels occupent surtout le centre de l'empire colonial français, la périphérie est aussi explorée et cartographiée par des voyageurs soucieux de transmettre un savoir géographique souvent inédit. Longtemps discréditée, la figure du baron de Lahontan (1666-1716) en est l'illustration parfaite. Débarqué au Canada en 1683, cet officier se rend célèbre par la publication des Nouveaux voyages dans l'Amérique septentrionale, récit qui fait la part belle aux cartes géographiques, "ce qu'il y a de plus utile et de très conforme au goût du siècle». Prévenant, Lahontan avertit l'explorateur qu'il doit se munir d'un astrolabe, d'un demi-cercle, de plusieurs boussoles, d'une pierre d'aimant, de deux grosses montres, de pinceaux, de couleurs et de papier à dessin pour réaliser ses journaux et ses cartes ${ }^{43}$. Celles que lui-même trace sont très instructives, permettant de voir « d'un coup d'œil la véritable disposition de ce pays-là ». Sa Carte générale de Canada présente à la fois les pays où les Indiens chassent le castor et les limites de la Nouvelle-France, du moins celles qu'il propose à ses lecteurs. Lui-même explorateur, Lahontan relate sa propre expédition sur le grand fleuve Mississippi. Empruntant un affluent inconnu des Français, il croise plusieurs bourgades indiennes densément peuplées. Très bien accueilli chez les Gnacsitares, Lahontan se fait offrir par le chef non seulement les femmes du village mais aussi une carte dessinée sur des peaux de cerfs. Trop fatigué et affamé, il refuse les femmes (dit-il!) mais accepte la carte, qu'il s'empressera de publier dans ses récits ${ }^{44}$. Cette fameuse Carte de la rivière Longue indique clairement jusqu'où l'expédition a pu se rendre avant de rebrousser chemin. La représentation gravée se présente sous forme de diptyque, où les deux tableaux sont séparés par une ligne centrale et une fleur de lys. Du côté est, le cartographe a représenté les territoires qu'il a explorés. Du côté ouest, il y a retranscrit la carte indienne. En marge figurent les représentations d'une maison et d'une embarcation des Tahuglauk. Peu importe que cette carte publiée soit vraie ou fausse; le seul fait de

43. Louis Armand de Lom d'Arce, baron de Lahontan, Nouveaux voyages de $M^{r}$ le baron de Lahontan, dans l'Amérique septentrionale, La Haye, Chez les frères L'Honoré, 1703, lettre XVI, datée du 28 mai 1689 et rédigée à Michillimackinac.

44. Carte de la rivière Longue et de quelques autres qui se dechargent dans le grand fleuve de Missisipi [sic], dans Nouveaux voyages de Louis Armand de Lom d'Arce, baron de Lahontan, La Haye, Frères l'Honoré, 1703, vol. 1. 
l'inclure dans le livre permet en quelque sorte d'authentifier la parole de l'explorateur, de la rendre crédible. Ici, l'acte de cartographier témoigne ou fait croire à une présence tangible sur le terrain, comme si l'auteur avait voulu se prémunir des attaques qui allaient fuser contre lui (pendant bien longtemps, pourrait-on ajouter ${ }^{45}$ ). Sans porter de jugement sur le dessin autochtone, au contraire, le baron de Lahontan explique que les Amérindiens utilisent les cartes en période de guerre et de chasse :

Quoique les Sauvages n'ayent aucune connoissance de la Geographie, non plus que des autre Sciences, ils font les Cartes du Monde les plus correctes des Païs qu'ils connoissent, auxquels il ne manque que les latitudes et les longitudes des lieux. Ils y marquent le vrai Nord, selon l'Etoile Polaire, les Ports, les Havres, les Rivieres, les Anses et les Côtes des Lacs, les Chemins, les Montagnes, les Bois, les Marais, les Prairies, $\&$ c. en comptant les distances par journées, demie journées de Guerriers, chaque journée valent cinq lieues. Ils font ces Cartes Chorographiques particuliers sur des écorces de bouleau, \& toutes les fois que les Anciens tiennent des Conseils de Guerre et de Chasse, ils ne manquent pas de les consulter ${ }^{46}$.

Si cet extrait renseigne à la fois sur les méthodes et les usages de la cartographie amérindienne, il permet aussi à Lahontan de décrire le Sauvage comme un homme attentif à son environnement, avide de connaissances géographiques, non pas théoriques mais bien utiles sur le terrain, reconnaissant ainsi l'universalité de la cartographie comme mode de communication.

À l'instar de Lahontan, d'autres explorateurs cartographes vont sillonner le continent dans toutes les directions pour permettre d'en prendre la pleine mesure et d'en affiner la description géographique. Dans les années 1730, le clan des La Vérendrye a produit plusieurs

45. Le premier à mettre en doute les données fournies par Lahontan est le géographe Guillaume Delisle sur sa Carte du Canada ou de la Nouvelle France publiée en 1703 où l'on peut lire que le baron a peut-être «inventé toutes ces choses ce qu'il est difficile de résoudre étant le seul qui a pénétré dans ces vastes contrées ». Cela ne l'empêche pas d'incorporer la rivière Longue dans le prolongement de la rivière Moingona (Des Moines). Pour un aperçu des polémiques sur le voyage de Lahontan, voir les Euvres complètes éditées par Réal Ouellet, Montréal, Presses de l'Université de Montréal, 1990.

46. Memoires de l'Amerique septentrionale, ou, La suite des voyages de Mr. le baron, La Haye, Chez les frères L'Honoré, 1704, p. 104-105. 
cartes qui sont parmi les premières à décrire les grandes plaines de l'Ouest américain. Comprenant l'importance des guides amérindiens pour explorer et traverser le continent, ils sollicitent l'aide d'un Amérindien dénommé Ochagach pour dessiner le réseau hydrographique à l'ouest des Grands Lacs, ce qu'il fait avec du charbon de bois frotté sur de l'écorce de bouleau. La carte originale est fort probablement perdue à jamais. Mais La Vérendrye prend la peine de reproduire le croquis sur papier et d'en faire part au secrétariat d'État de la Marine comme preuve documentaire ${ }^{47}$. Même si cette carte ne correspond pas aux standards de la cartographie européenne, elle répond aux besoins des voyageurs en mettant en valeur et en amplifiant les éléments les plus importants: lacs, cours d'eau et portages présentés en enfilade. L'échelle de la carte est ainsi proportionnelle à l'expérience vécue, soit au temps de parcours, plutôt qu'aux distances absolues. C'est une carte utilitaire et linéaire, qui montre un chemin à parcourir pour se rendre d'un point $\mathrm{A}$ à un point $\mathrm{B}$, à travers les contrées de divers peuples amérindiens.

La diffusion de cette carte suscite un engouement pour la description de l'Amérique dans les milieux scientifiques. Philippe Buache la reproduit dans un document publié sous les auspices de l'Académie des sciences. Alors qu'auparavant on taisait habituellement les sources amérindiennes, elles sont dorénavant mises en valeur, semblant conférer une plus grande crédibilité à la description, l'autochtone étant le mieux placé pour décrire la géographie de son pays. Le cartographe Jacques Nicolas Bellin a lui aussi entre les mains l'œuvre d'Ochagach. Son défi consiste à intégrer cette carte pleine de promesses à ses synthèses cartographiques, ce à quoi il parvient avec quelques difficultés. Ceux qui se fient à son œuvre cartographique ont lieu d'être optimistes: les La Vérendrye ne sont pas loin de leur but. En vérité, ils sont à environ 2000 kilomètres (à vol d'oiseau) de la côte du Pacifique.

\section{Cartographie cadastrale}

Lune des propositions de Franquelin consistait à cartographier les terres défrichées conformément au "papier terrier», registre officiel dans lequel on compilait les descriptions détaillées des seigneuries, des

47. Il existe quelques copies de cette carte, notamment deux cartes manuscrites au SHD, rec. 67, p. 16, et rec. 67, p. 87. 
censives et des redevances seigneuriales. Dès 1678, sur commande de l'intendant Duchesneau, il avait dessiné une carte à petite échelle localisant les seigneuries du Canada ${ }^{48}$. Destinée au ministre Colbert, cette carte, même imprécise, devait aider à faciliter la lecture du papier terrier. Mais elle demeurait approximative et il fallait d'autres cartes à plus grande échelle pour connaître les limites des seigneuries et en faciliter l'administration.

Une trentaine d'années plus tard, l'arpenteur et sous-officier Gédéon de Catalogne envisage et met à exécution un programme d'envergure qui comble les lacunes en ce domaine. En 1707, il remet à l'intendant Raudot un plan de l'île de Montréal avec toutes ses concessions ${ }^{49}$. Il propose ensuite d'étendre la description à toutes les autres seigneuries de la colonie. Encouragé par le ministre, il visite pendant deux ans toutes les côtes du pays, par les temps les plus rudes et les plus difficiles. Heureusement pour lui, Catalogne n'est pas seul pour accomplir cette tâche gigantesque. Les documents d'archives mentionnent la présence d'un certain sieur Robert pour l'appuyer, puis celle de Jean Baptiste de Couagne, fils d'un riche marchand montréalais ${ }^{50}$. Celui-ci reçoit sa commission d'arpenteur et de mesureur royal en 1708, à la recommandation des Sulpiciens, seigneurs de Montréal. Lannée suivante, il signe avec Catalogne les cinq feuillets de grand format (environ $125 \times$ $170 \mathrm{~cm}$ ) couvrant les régions de Québec et de Trois-Rivières, début remarquable dans la profession! Ces cartes dépeignent à merveille le découpage du territoire en concessions tout en consignant les noms de tous les censitaires chefs de famille. On peut imaginer la complexité de ce travail laborieux et coûteux mais tout de même appuyé par l'État. D’une indéniable utilité administrative, les cartes permettaient de situer la population, d'en assurer le contrôle et de mieux planifier le développement de la colonie. Leur justesse ayant été mise en cause par un autre ingénieur, elles sont exposées au palais de l'intendant pendant

48. J. B. L. Franquelin, Carte pour servir a l'eclaircissement du papier terrier de la Nouvelle France [Québec], 1678. BNF, Rés. SH Archives nº 23 B.

49. Résumé d’une lettre de Catalogne, 10 novembre 1707. ANOM, COL C11A 27 / fol. 210.

50. De Couagne a probablement été élevé bien jeune dans l'amour et le respect du savoir historique et géographique puisque son père lit des œuvres historiques et tapisse sa chambre de cartes géographiques. Voir Louise Dechêne, Habitants et marchands de Montréal au XVII eiècle, Paris, Plon, 1974, p. 391. 
15 jours, «à la censure de tout le monde ${ }^{51}$ ». On rapporte alors que plusieurs personnes en font faire des copies, ce qui fait valoir leur utilité et leur utilisation en milieu colonial.

\section{Une hypothèse de recherche: pouvoir et savoir en milieu colonial}

Ces quelques exemples puisés à même le vaste corpus de la cartographie coloniale montrent l'intérêt marqué de l'État pour le savoir géographique, surtout à partir des années 1680. La carte devient alors un vecteur de communication par excellence entre l'explorateur et l'administration locale, entre l'administration locale et la cour du roi et même entre le voyageur écrivain et son lecteur, comme l'illustre l'exemple du baron de Lahontan. On assiste alors à une véritable ébullition de la chose cartographique qui s'explique non seulement par la présence d'une constellation de professionnels établis mais aussi par la sensibilité accrue des acteurs politiques à l'utilité du langage cartographique pour gouverner. Le marquis de Denonville, gouverneur pendant seulement quatre ans, semble plus que tout autre disposé à encourager le travail des cartographes. En contribuant à l'accroissement et à la circulation du savoir géographique, il amorce ainsi un mouvement favorable au développement des sciences dans la colonie. Mais pour que le mouvement prenne son élan, le soutien actif de la métropole est indispensable. Fraîchement nommé secrétaire d'État à la Marine, le marquis de Seignelay autorise la création des postes clés d'ingénieurs et d'hydrographes du roi, grâce auxquels il consolide la production d'un savoir colonial utile à l'État. Dès lors, pour tous les intendants et gouverneurs qui se sont succédé à Québec, il était essentiel de se donner les outils nécessaires à une meilleure connaissance du territoire ainsi qu'à une meilleure communication avec les autorités métropolitaines ${ }^{52}$. Qu'il

51. Lettre des intendants Raudot au ministre, 14 novembre 1709. ANOM COL $\mathrm{C}_{11} \mathrm{~A}_{30} /$ fol. 271-320v.

52. À cet égard, il ne faut pas non plus négliger l'usage des recensements et des papiers terriers, dont il n’a pas été question dans le présent article mais qui constituent des outils indispensables pour bien apprécier l'état du peuplement et du développement de la colonie. Il peut ainsi être utile de rappeler que l'intendant Talon fit le premier usage des recensements dès 1666 et que ceux-ci furent ensuite utilisés relativement souvent (du moins plus fréquemment qu'en France) par les intendants qui se sont succédé à la tête de la colonie. 
s'agisse d'hydrographie, de topographie, d'arpentage ou d'exploration, la connaissance géographique était indispensable non seulement au maintien mais aussi à l'accroissement de la colonie et de son commerce.

Les exemples mentionnés dans cet article ne représentent qu'un faible fragment des sources disponibles. En effet, il faut souligner l'importance du corpus constitué de centaines, voire de milliers de documents cartographiques dispersés parmi plusieurs institutions. Ces documents ont été inventoriés et étudiés à des degrés variables mais souvent de façon fragmentaire, sans véritable vue d'ensemble. C'est pourquoi la constitution d'un vaste catalogue collectif s'avère un travail préalable incontournable, rassemblant dans un lieu virtuel unique l'ensemble des cartes de la Nouvelle-France aujourd'hui conservées en Europe et en Amérique du Nord ${ }^{53}$.

Une fois ce vaste ensemble reconstitué, il sera plus facile de confirmer ou d'infirmer l'hypothèse d'une rupture marquée dans les années 1680 et, assurément, d'en déterminer les causes. Mis en perspective avec les autres espaces coloniaux (notamment les Amériques britannique et espagnole), les résultats de recherche pourront confirmer ou non l'adéquation entre l'intensification de l'acte cartographique et le mouvement de centralisation des décisions politiques, vérifiant si l'intérêt pour la chose cartographique demeure égal jusqu’à la fin du régime, en 176o. Abordant son objet sous plusieurs angles (l'étude des cartographes, des destinataires, des réseaux de circulation du savoir, des usages de la cartographie, de l'intégration du savoir indigène à la sphère scientifique française, de la rhétorique des cartes et plans, etc.), cette enquête devra permettre de mieux comprendre le rôle de l'État comme principal organisateur et utilisateur des savoirs, de mieux saisir, en somme, les rapports entre pouvoir et sciences dans un contexte de colonisation et d'appropriation du territoire.

53. Fruit d'un partenariat entre Bibliothèque et Archives nationales du Québec et la Bibliothèque nationale de France, ce catalogue actuellement en préparation vise à rassembler virtuellement l'ensemble des cartes de la Nouvelle-France, fortifiant ainsi la recherche sur le territoire québécois et, par extension, sur le territoire nordaméricain. 